\title{
Efeito de repelência do inseticida deltamethrin sobre insetos de raças resistentes e suscetíveis de Rhyzopertha dominica (F.) (Coleoptera, Bostrichidae) em grãos de trigo armazenado ${ }^{1}$
}

\author{
Helenara Beckel², Irineu Lorini ${ }^{3} \&$ Sonia M. N. Lazzari ${ }^{4}$
}

${ }^{1}$ Contribuição no 1451 do Departamento de Zoologia, Universidade Federal do Paraná. Parte da dissertação de Mestrado em Entomologia do $1^{\circ}$ autor, Convênio Embrapa Trigo-UFPR.

${ }^{2}$ R. 20 de Setembro, 359, 99025-580, Passo Fundo-RS. Bolsista do CNPq. helenara@via-rs.net

${ }^{3}$ Embrapa Trigo-Centro Nacional de Pesquisa de Trigo. Caixa Postal 451, 99001-970, Passo Fundo-RS. ilorini@cnpt.embrapa.br

${ }^{4}$ Departamento de Zoologia, Universidade Federal do Paraná. Caixa Postal 19020, 81531-990, Curitiba-PR. Bolsista do CNPq. lazzari@ufpr.br

\begin{abstract}
Repellency of deltamethrin against resistant and susceptible insects of strains of Rhyzopertha dominica (F.) (Coleoptera, Bostrichidae) in stored wheat. This research was carried out in order to study the effect of repellency of the insecticide deltamethrin against the lesser grain borer, Rhyzopertha dominica (F.). Four strains of $R$. dominica were used: two of them were deltamethrin resistant, BR6 and BR12, and two were susceptible, BR4 and UK1. The insects were collected in storage units and maintained in laboratory. One hundred insects were released in the bottom of a plastic pot containing untreated wheat grain connected to another pot of same size containing wheat grain treated with $\mathrm{LC}_{5}$, $\mathrm{LC}_{25} \mathrm{LC}_{50}$, separately, for each strain, replicated four times. The pots were kept horizontally in order to let the insects to move freely between the two compartments, at $25 \pm 1{ }^{\circ} \mathrm{C}$ and $60 \pm 5 \%$ of temperature and relative humidity, respectively. The assessment of insect distribution in the pots was performed 12 days after the releasing. The insects avoided the treated grain at all concentrations, meaning that the insecticide presents an effect of repellency against the insects. Nevertheless, there were no significant differences in the number of individuals of the susceptible strains between the treatments and the control. On the other hand, the resistant strains showed higher response to deltamethrin than the susceptible insects, especially at higher concentrations. The study of such behavior is fundamental for decision making in resistance management programs of stored product insects.
\end{abstract}

KEYWORDS. Behavior; Insecta; lesser grain borer; resistance; stored grains.

RESUMO. Efeito de repelência do inseticida deltamethrin sobre insetos de raças resistentes e suscetíveis de Rhyzopertha dominica (F.) (Coleoptera, Bostrichidae) em grãos de trigo armazenado. Rhyzopertha dominica (F.) foi submetida a estudos de repelência ao inseticida deltamethrin aplicado em grãos de trigo, em laboratório. Foram testadas quatro raças de $R$. dominica, duas resistentes ao deltamethrin, BR6 e BR12, e duas suscetíveis, BR4 e UK1, coletadas em unidades armazenadoras de grãos e mantidas em multiplicação em laboratório. $\mathrm{O}$ experimento foi realizado em quatro repetições, liberando-se 100 insetos, por repetição, em um pote de plástico contendo grãos de trigo não tratados; esse pote ficava conectado a outro de mesmo tamanho contendo grãos de trigo tratados com $\mathrm{CL}_{5}, \mathrm{CL}_{25} \mathrm{e} \mathrm{CL}_{50}$ do inseticida deltamethrin. separadamente, para cada raça. O conjunto foi mantido na posição horizontal, de forma a permitir a livre passagem dos insetos entre os dois compartimentos, a $25 \pm 1^{\circ} \mathrm{C}$ e $60 \pm 5 \%$ de temperatura e de umidade relativa do ar, respectivamente. A avaliação da distribuição dos insetos no interior dos potes ocorreu 12 dias após sua liberação. Os insetos evitaram os grãos tratados com todas as concentrações de deltamethrin, indicando o efeito de repelência que o inseticida exerce sobre eles. No caso das raças suscetíveis, a diferença não foi significativa entre o número de indivíduos nas porções tratadas e não tratadas, nas diferentes concentrações. Por outro lado, os indivíduos das raças resistentes apresentaram um comportamento de repelência significativamente mais acentuado que os das suscetíveis, especialmente nas concentrações mais elevadas. O estudo dessas respostas comportamentais é fundamental para a tomada de decisões em programas de manejo da resistência de insetos de produtos armazenados.

PALAVRAS-CHAVE. Besourinho-dos-cereais; comportamento; grãos armazenados; Insecta; resistência.

O inseticida deltamethrin é um dos mais importantes piretróides recomendados como protetores de grãos (Lorini \& Galley 1996; 1999). O estudo do efeito repelente de deltamethrin sobre Rhyzopertha dominica (Fabricius, 1792) (Coleoptera, Bostrichidae) é fundamental para controle dessa espécie, uma vez que a ação repelente, por contato ou inalação, pode reduzir a probabilidade de infestação no grão (Hussain \& Mondal 1994).

Collins et al. (1988) indicaram que o conhecimento dos efeitos repelentes de compostos químicos permite o emprego de taxas de aplicação de inseticidas substancialmente reduzidas, com conseqüente redução na presença de resíduos químicos em grãos e derivados e nos custos de controle, além de acentuada inibição de infestações posteriores devido às propriedades repelentes do protetor de grãos.

O conhecimento do comportamento das pragas em relação aos inseticidas vem contribuindo significativamente para a compreensão da reincidência de insetos na massa de grãos, logo após o tratamento químico e sua sobrevivência em produtos armazenados. Prickett \& Ratcliffe (1977), estudando 
adultos de Tribolium castaneum (Herbst, 1797) (Coleoptera, Tenebrionidae), observaram que os insetos evitaram superfícies tratadas com pyrethrins, com bioresmethrin, com DDT e com lindane, mas não superfícies tratadas com inseticidas organofosforados. Segundo Collins et al. (1988), inseticidas que agem diretamente sobre o sistema nervoso central ou nervos periféricos (DDT e grupos piretróides) podem, provavelmente, irritar mais ou repelir coleópteros de produtos armazenados.

Este trabalho visou determinar, em laboratório, o efeito de repelência do inseticida piretróide deltamethrin sobre adultos de raças resistentes e suscetíveis de $R$. dominica em grãos de trigo.

\section{MATERIAL E MÉTODOS}

Insetos adultos de quatro raças foram usados no experimento. As raças BR4, BR6 e BR12 foram coletadas em armazéns de trigo no Rio Grande do Sul em 1994, 1997 e 1998, respectivamente. Indivíduos da raça UK1 são originários da Inglaterra, Reino Unido, onde se encontram em multiplicação em laboratório há mais de 15 anos. Uma amostra dessa raça foi trazida ao Brasil, em 1997, para os trabalhos de resistência desenvolvidos na Embrapa Trigo, RS.

Indivíduos das raças BR4 e UK1 são suscetíveis, e indivíduos das raças BR6 e BR12, resistentes ao deltamethrin (Lorini \& Galley 1999, Beckel et al. 1999). Os insetos das diferentes raças, desde a época da coleta, foram mantidos em multiplicação no Laboratório de Entomologia da Embrapa Trigo e, em virtude disso, receberam as seguintes designações: geração $\mathrm{F}_{1}$, indicando a primeira geração de laboratório e criada sem seleção pelo inseticida, e $\mathrm{F}^{\mathrm{d}}$, representando as gerações de raças mantidas em laboratório sob a seleção do inseticida.

Insetos adultos, não sexados, das raças resistentes BR6 (geração $\mathrm{F}_{18}^{\mathrm{d}}$ ) e BR12 (geração $\mathrm{F}_{6}$ ) e das raças suscetíveis BR4 e UK1 (geração $\mathrm{F}_{28}$ ), com 20-30 dias de idade, foram usados para o experimento. $\mathrm{O}$ delineamento experimental foi de blocos ao acaso, em quatro repetições, e os tratamentos foram $\mathrm{CL}_{5}$, $\mathrm{CL}_{25}$ e $\mathrm{CL}_{50}$ (concentrações letais que causam 5\%, 25\% e 50\% de mortalidade da população) e uma testemunha sem inseticida para cada raça. Cada unidade experimental consistiu de dois potes de plástico, com capacidade de $250 \mathrm{~mL}$, preenchidos com $240 \mathrm{~g}$ de grãos de trigo, justapostos e conectados entre si com uma fita adesiva.

Foram liberados 100 insetos adultos, por repetição, em um dos potes, que continha grãos de trigo não tratados. No outro pote foram colocados grãos de trigo tratados com $\mathrm{CL}_{5}, \mathrm{CL}_{25} \mathrm{e}$ $\mathrm{CL}_{50}$ do inseticida deltamethrin. Na montagem do conjunto, os potes com grãos tratados foram invertidos sobre os potes com grãos não tratados, com auxílio de uma lâmina de metal, retirada posteriormente. Após a infestação, os potes foram mantidos na posição horizontal, possibilitando o pleno fluxo de insetos entre os potes, em sala climatizada com temperatura de $25 \pm 1^{\circ} \mathrm{C}$ e umidade relativa do ar de $60 \pm 5 \%$.

$\mathrm{O}$ experimento foi avaliado 12 dias após a liberação dos insetos, por peneiração dos grãos (peneira com malha $\mathrm{n}^{\circ} 20$, abertura $850 \mathrm{~mm}$ ) e contagem do número de insetos vivos e mortos, em cada pote.

A determinação prévia de $\mathrm{CL}_{5}, \mathrm{CL}_{25}$ e $\mathrm{CL}_{50}$ foi obtida por meio de bioensaios de laboratório, analisados pelo programa estatístico GLIM, versão 3.77 (Crawley 1993), e os resultados da contagem de insetos foram submetidos à analise de variância, e as médias comparadas entre si, pelo teste de Tukey, a $5 \%$ de probabilidade.

\section{RESULTADOS EDISCUSSÃO}

Os resultados do efeito de repelência de deltamethrin sobre $R$. dominica, em grãos de trigo, mostraram diferenças significativas entre as raças suscetíveis e as resistentes. Pôdese observar também, após a peneiração dos grãos, que alguns insetos penetraram nos grãos de trigo e estes foram excluídos da contagem (Tabela I).

$\mathrm{Na}$ raça suscetível BR4, os insetos não apresentaram diferenças significativas, em relação ao controle, em nenhuma das CLs testadas. Já na raça UK1 houve menor número de insetos nos potes tratados no controle e na $\mathrm{CL}_{50}$, os quais foram inferiores à $\mathrm{CL}_{5}$ e à $\mathrm{CL}_{25}$. A raça resistente $\mathrm{BR} 6$ apresentou menor número de insetos nos potes contendo grãos tratados, diferindo significativamente do controle sem inseticida, na $\mathrm{CL}_{25}$ e na $\mathrm{CL}_{50}$, os quais não diferiram entre si. (Tabela I). Na raça resistente BR12, o número de insetos sobre os grãos tratados foi significativamente mais baixo que nos grãos não tratados, e essa diferença acentuou-se com a elevação da $\mathrm{CL}_{5}$ para a $\mathrm{CL}_{50}$ (Tabela I), indicando o efeito de repelência que o produto apresenta sobre essa raça resistente.

Os resultados encontrados para insetos da raça resistente BR6, e mais acentuadamente para os de BR12, corroboram o trabalho de Pinniger (1974), que, estudando a espécie $T$. castaneum, em laboratório, demonstrou que a sobrevivência dos insetos pode ser atribuída à fuga do contato com o inseticida, permanecendo esses mais tempo em refúgios não contaminados contendo alimento. Em outros estudos, $R$. dominica (Laudani \& Swank 1954) e Sitophilus oryzae (Linnaeus, 1763) (Coleoptera, Curculionidae) (Chadwick 1962) foram repelidos por grãos tratados com pyrethrin, embora tratamentos com deltamethrin tenham falhado em restringir a invasão de $R$. dominica em grãos tratados (Lorini \& Galley 1998), contrastando com os resultados encontrados neste trabalho.

Estudos de Collins et al. (1988) indicaram que um conhecimento mais amplo dos efeitos repelentes de compostos químicos pode contribuir para reduzir o nível de aplicação de inseticidas, diminuindo, conseqüentemente os resíduos químicos em grãos e subprodutos e os custos de controle e, ainda, inibindo infestações posteriores devido às propriedades repelentes do protetor de grãos.

Muitos aspectos comportamentais têm sido estudados, mas o comportamento de repelência de $R$. dominica em grãos tratados com inseticida pode estar envolvido no mecanismo de resistência da praga. O estudo das alterações de comportamento em relação à resistência das pragas aos 
Tabela I. Número médio ( \pm erro padrão) de adultos de $R$. dominica (raças BR4, UK1, BR6 e BR12) presentes em grãos de trigo tratados com as concentrações $\mathrm{CL}_{5} \mathrm{CL}_{25}$ e $\mathrm{CL}_{50}$ de deltamethrin e sem tratamento. Média de 100 insetos/pote de plástico contendo $240 \mathrm{~g}$ de grãos, em quatro repetições, a $25 \pm 1^{\circ} \mathrm{C}$ e UR de $60 \pm 5 \%$, Passo Fundo, RS, 1999.

\begin{tabular}{|c|c|c|c|c|c|c|c|c|}
\hline \multirow{2}{*}{$\begin{array}{l}\text { Raça/ } \\
\text { Concentração }\end{array}$} & \multicolumn{2}{|c|}{ BR4 } & \multicolumn{2}{|c|}{ UK1 } & \multicolumn{2}{|c|}{ BR6 } & \multicolumn{2}{|c|}{ BR12 } \\
\hline & $\mathrm{s} /$ trat. $^{1}$ & c/trat. ${ }^{2}$ & s/trat. ${ }^{1}$ & c/trat. ${ }^{2}$ & s/trat. ${ }^{1}$ & $\mathrm{c} /$ trat. $^{2}$ & $\mathrm{~s} /$ trat. $^{1}$ & c/trat. ${ }^{2}$ \\
\hline $\begin{array}{l}\text { Sem } \\
\text { Inseticida }\end{array}$ & $\begin{array}{l}53,2 \mathrm{aA} \\
( \pm 0,38)\end{array}$ & $\begin{array}{c}15,2 \mathrm{aB} \\
( \pm 0,47)\end{array}$ & $\begin{array}{l}50,2 \mathrm{aA} \\
( \pm 0,56)\end{array}$ & $\begin{array}{r}19,0 \mathrm{bB} \\
( \pm 0,50)\end{array}$ & $\begin{array}{l}47,5 \mathrm{bA} \\
( \pm 0,27)\end{array}$ & $\begin{array}{r}34,0 \mathrm{aB} \\
( \pm 0,56)\end{array}$ & $\begin{array}{l}53,2 \text { bA } \\
( \pm 0,33)\end{array}$ & $\begin{array}{r}19,2 \mathrm{aB} \\
( \pm 0,58)\end{array}$ \\
\hline $\mathrm{CL}_{5}$ & $\begin{array}{l}60,7 \mathrm{aA} \\
( \pm 0,24)\end{array}$ & $\begin{array}{r}15,7 \mathrm{aB} \\
( \pm 0,84)\end{array}$ & $\begin{array}{l}43,2 \mathrm{aA} \\
( \pm 0,76)\end{array}$ & $\begin{array}{r}34,5 \mathrm{aA} \\
( \pm 0,61)\end{array}$ & $\begin{array}{l}59,5 \mathrm{abA} \\
( \pm 0,37)\end{array}$ & $\begin{array}{r}31,5 \mathrm{aB} \\
( \pm 0,36)\end{array}$ & $\begin{array}{l}73,0 \mathrm{aA} \\
( \pm 0,48)\end{array}$ & $\begin{array}{c}8,7 \mathrm{bB} \\
( \pm 0,51)\end{array}$ \\
\hline $\mathrm{CL}_{25}$ & $\begin{array}{l}58,0 \mathrm{aA} \\
( \pm 0,22)\end{array}$ & $\begin{array}{r}17,0 \mathrm{aB} \\
( \pm 0,47)\end{array}$ & $\begin{array}{l}43,5 \mathrm{aA} \\
( \pm 0,76)\end{array}$ & $\begin{array}{r}31,5 \mathrm{aB} \\
( \pm 0,52)\end{array}$ & $\begin{array}{l}70,0 \mathrm{aA} \\
( \pm 0,49)\end{array}$ & $\begin{array}{r}17,5 \mathrm{bB} \\
( \pm 0,86)\end{array}$ & $\begin{array}{l}81,2 \mathrm{aA} \\
( \pm 0,33)\end{array}$ & $\begin{array}{c}2,7 \mathrm{cB} \\
( \pm 0,58)\end{array}$ \\
\hline $\mathrm{CL}_{50}$ & $\begin{array}{l}68,5 \mathrm{aA} \\
( \pm 0,31)\end{array}$ & $\begin{array}{c}15,2 \mathrm{aB} \\
( \pm 0,48)\end{array}$ & $\begin{array}{l}54,2 \mathrm{aA} \\
( \pm 0,22)\end{array}$ & $\begin{array}{r}17,5 \mathrm{bB} \\
( \pm 0,44)\end{array}$ & $\begin{array}{l}63,5 \mathrm{aA} \\
( \pm 0,25)\end{array}$ & $\begin{array}{r}26,7 \mathrm{abB} \\
( \pm 0,62)\end{array}$ & $\begin{array}{l}78,2 \mathrm{aA} \\
( \pm 0,14)\end{array}$ & $\begin{array}{c}2,5 \mathrm{cB} \\
( \pm 0,55)\end{array}$ \\
\hline
\end{tabular}

${ }^{1}$ Número de insetos no pote onde ocorreu a liberação inicial, com grãos de trigo não tratados.

${ }^{2}$ Número de insetos recuperados no pote onde não ocorreu a liberação inicial, com grãos de trigo tratados.

Médias seguidas de mesmas letras, dentro da mesma raça, minúsculas na coluna e maiúsculas na linha, não diferem significativamente entre si, pelo teste de Tukey, a $5 \%$ de probabilidade.

inseticidas precisa ser mais bem compreendido para determinar o quanto estas podem explicar a resistência de pragas de grãos armazenados e, ainda, para aplicar essa informação nas decisões com relação ao manejo da resistência.

\section{REFERÊNCIAS}

Beckel, H.; I. Lorini \& S. M. N. Lazzari. 1999. Hábito de deslocamento de raças resistentes e suscetíveis de Rhyzopertha dominica (F.) (Coleoptera; Bostrychidae) em superfície tratada com o inseticida deltamethrin, p.242-247, vol. 2. In: I Conferência Brasileira de Pós-Colheita, ed. Embrapa Trigo. Rio Grande do Sul, Porto Alegre.

Chadwick, P. R. 1962. Studies on the sub-lethal effects of pyrethrins on the grain weevil, Calandra oryzae L. Pyrethrum Post 6: 2026.

Collins, P. J.; E. R. Sinclair; C. J. Howitt \& R. L. Haddrell. 1988. Dispersion of grain beetles (Coleoptera) in grain partially treated with insecticide. Journal of Economic Entomology 81: 18101815.

Crawley, M. J. 1993. Glim for ecologists. Blackwell Scientific Publications. Oxford, United Kingdom, 379 p.
Hussain, M. M. \& K. A. M. S. H. Mondal. 1994. Repellent effect of nogos on adult Tribolium confusum D. and Rhyzopertha dominica F. Pakistan Journal of Zoology 26: 187-189.

Laudani, H. \& G. R. Swank. 1954. A laboratory apparatus for determining repellency of pyrethrum when applies to grain. Journal of Economic Entomology 47: 1105-1107.

Lorini, I. \& D. J. Galley. 1996. Changes in resistance status of Rhyzopertha dominica (F.) (Coleoptera: Bostrychidae), a pest of stored grain in Brazil, with and without deltamethrin selection. Resistant Pest Management Newsletter 8: 12-14.

Lorini, I. \& D. J. Galley. 1998. Relative effectiveness of topical, filter paper and grain applications of deltamethrin, and associated behaviour of Rhyzopertha dominica (F.) strains. Journal of Stored Products Research 34: 377-383.

Lorini, I. \& D. J. Galley. 1999. Deltamethrin resistance in Rhyzopertha dominica (F.) (Coleoptera: Bostrychidae), a pest of stored grain in Brazil. Journal of Stored Products Research 35: 37-45.

Pinniger, D. B. 1974. A laboratory simulation of residual populations of stored product pests and an assessment of their susceptibility to a contact insecticide. Journal of Stored Products Research 10: $217-223$

Prickett, A. J. \& C. A. Ratcliffe. 1977. The behaviour of Tribolium castaneum (Herbst) and Sitophilus granarius (L.) in the presence of insecticide treated surfaces. Journal of Stored Products Research 13: 145-148. 